\title{
HISTORIA RECIENTE DE LA UNIVERSIDAD DE ALCALÁ
}

\section{RECENT HISTORY OF THE UNIVERSITY OF ALCALA}

\section{AUTORES}

José Morilla Critz: Catedrático de Universidad. Área de Fundamentos de la Economía. jose.morilla@aol.com

\section{CURRÍCULUM VITAE}

Profesor en la Facultad de Ciencias Económicas y Empresariales. Universidad de Alcalá.

\section{RESUMEN}

Aunque pueda resultar extraño a cualquier pensamiento ordenado, hemos de comenzar diciendo que la apertura de la actual Universidad de Alcalá no fue, para aquellas instancias que tenían la capacidad de decidir al respecto, un proyecto de recuperación de la antigua Universidad. Más aún, también es difícil determinar cuando se tomó una decisión clara y definida de abrir de nuevo una Universidad en Alcalá de Henares por parte de las autoridades educativas españolas. Como veremos a continuación, el renacimiento de la Universidad de Alcalá en el siglo XX es, más que un hecho susceptible de datar, un proceso de confluencia de circunstancias que se van produciendo y entrelazando desde 1965.

En la historia que sigue no es fácil encontrar un agente refundador. Solamente algunos grupos de alcalaínos y, en ciertos casos, la institución municipal, tuvieron claro que Alcalá de Henares debía aprovechar cualquier oportunidad que se presentara para recuperar su carácter universitario. 


\title{
PALABRAS CLAVE
}

Universidad de Alcalá - Renacimiento - Historia

\begin{abstract}
Although it may seem strange to any ordered thinking, we must start by saying that the opening of the current University of Alcalá was not for those instances that have the capacity to decide on a project to recover the old University. Moreover, it is also difficult to determine when a decision was clear and definite to reopen a university in Alcalá de Henares by the Spanish educational authorities. As outlined below, the rebirth of the University of Alcalá in the twentieth century is more than a fact capable of dating, a process of confluence of circumstances that are produced and weaving since 1965.
\end{abstract}

In the story that follows is not easy to find an agent re-founder. Only a few groups of Alcalá and in some cases, the municipal institution, were clear that Alcala de Henares should take every opportunity that presented itself to regain its university status.

\section{KEY WORDS}

University of Alcalá - Renaissance - History

\section{ÍNDICE}

Prólogo (Presentación del estudio que publicamos)

1. El renacimiento de la Universidad de Alcalá (Por José Morilla Critz)

1.1. Primera parte: génesis de la resurrección 


\subsubsection{Introducción}

1.1.2. Los primeros pasos

1.1.3. Escuela Nacional de Administración Pública

\section{Prólogo}

Nuestra pretensión al abrir una sección histórica sobre nuestra Universidad es contarles a partir de este número nuestra historia pasada y reciente. Hemos pensado que sería mejor comenzar por la resurrección, porque sólo así se podrá entender la más reciente. Emplazamos a nuestros compañeros historiadores para que nos vayan remitiendo sus colaboraciones a fin de enriquecer nuestro conocimiento sobre nosotros mismos y nuestros antepasados.

Sin embargo, nos cabe la duda de que quizás deberíamos titular esta sección "historia de la Universidad de Alcalá" simplemente, ya que por más que se quiera, y por mucho que recuperemos el patrimonio inmobiliario de la antigua Universidad Complutense, jamás seremos la continuación de aquella. Prueba de ello es que la gran biblioteca trasladada a Madrid, tras el cierre en el siglo XIX, es ahora parte integrante del patrimonio de la Universidad madrileña. Por cierto que hará apenas 10 años que dicha biblioteca fue restaurada y microfilmada, sin que nuestras autoridades intentaran siquiera hacerse con una copia de las microfichas. Pero eso sería el tema de otros artículos y no queremos entrar aquí a discutir el porqué de esa desidia.

Lo que a partir de este número presentamos, en una serie de capítulos, es el texto escrito por nuestro compañero José Morilla Critz, Catedrático de la Facultad de Económicas y pionero del llamado, por él mismo, "Renacimiento de la Universidad de Alcalá". Este trabajo fue publicado por El Colegio Oficial de Arquitectos de 
Madrid y la propia Universidad de Alcalá, en 1990, como parte de un libro titulado La Universidad de Alcalá I, de tirada muy reducida y al que sólo tuvieron acceso algunos privilegiados. La historia contada por José Morilla consta de más de 70 páginas de letra apretada, razón por la cual hemos decidido publicarla por entregas, para hacerla más adecuada al formato electrónico de Vivat Academia y mantener además la atención del lector en los próximos meses, sin producirle un empacho de datos.

Como todos ustedes saben, una de nuestras intenciones es dar a conocer nuestra Universidad, en primer lugar a la propia comunidad universitaria alcalaína, y además a todos aquellos que, desde el exterior, se han convertido en lectores habituales. Nada mejor para conseguirlo que difundir este trabajo exhaustivo y completo, que comienza con los prolegómenos de la reconversión de Alcalá en ciudad universitaria, cuando era una ciudad militar, con incipiente industrialización, sede de la llamada Universidad Laboral, (alimentada ésta con estudiantes procedentes de toda la geografía española). La historia termina en una fecha en que el número de alumnos era de aproximadamente 13000, número óptimo, según los cánones de la Universidad media europea, en palabras del propio Morilla. A partir de ahí habrá que escribir la historia más reciente, que, como se verá, se ha desviado bastante de las intenciones primeras, por las que un gran número de universitarios luchamos en épocas pretéritas. Algunos de aquellos nos han dejado por voluntad propia, posiblemente porque tenían ofertas más atractivas que las proporcionadas por nuestra Institución. Otros nos han dejado definitivamente, porque la vida para algunos es demasiado corta, vaya para ellos nuestro cariñoso recuerdo. Muchos, afortunadamente, quedamos luchando, codo con codo de recursos humanos más jóvenes, convencidos de que aún podemos recuperar Alcalá como una Universidad de calidad y no sólo como una Universidad inmobiliaria. 


\section{El renacimiento de la Universidad de Alcalá (Por José Morilla Critz)}

\subsection{Primera parte: génesis de la resurrección}

\subsubsection{Introducción}

Aunque pueda resultar extraño a cualquier pensamiento ordenado, hemos de comenzar diciendo que la apertura de la actual Universidad de Alcalá no fue, para aquellas instancias que tenían la capacidad de decidir al respecto, un proyecto de recuperación de la antigua Universidad. Más aún, también es difícil determinar cuando se tomó una decisión clara y definida de abrir de nuevo una Universidad en Alcalá de Henares por parte de las autoridades educativas españolas. Como veremos a continuación, el renacimiento de la Universidad de Alcalá en el siglo XX es, más que un hecho susceptible de datar, un proceso de confluencia de circunstancias que se van produciendo y entrelazando desde 1965.

En la historia que sigue no es fácil encontrar un agente refundador. Solamente algunos grupos de alcalaínos y, en ciertos casos, la institución municipal, tuvieron claro que Alcalá de Henares debía aprovechar cualquier oportunidad que se presentara para recuperar su carácter universitario.

Fuera de Alcalá de Henares, un tema que hoy día nos puede parecer de una indudable importancia (recuperar una Universidad y el entorno urbano y arquitectónico que representó en su tiempo) no fue objeto de atención casi hasta nuestros días. En el siglo XIX uno de los Gobiernos de Narváez, de 1867, expresó el deseo de reabrir la Universidad de Alcalá, proyecto que muy pronto se abandonó, antes ya de la caída del 
breve gabinete, entre una fuerte oposición de la intelectualidad y la prensa. La idea apareció otra vez fugazmente, en 1922, como petición al Gobierno de Primo de Rivera, por parte de 20 catedráticos de la Universidad Central de Madrid.

En Alcalá de Henares, la utopía de la recuperación de la Universidad fue a lo largo de los siglos XIX y XX un elemento de unión entre todos los alcalaínos. Su primera manifestación fue la constitución de la "Sociedad de Condueños", caso único en España; también se manifestó en la confluencia de tendencias políticas, primero entre liberales y conservadores, después entre los partidos del sistema y el republicanismo y el socialismo, más adelante con el franquismo y, finalmente, en la democracia. En la historia reciente es interesante constatar cómo los programas municipales de todas las opciones políticas, desde 1978, recogieron la idea de recuperar el carácter universitario de Alcalá y potenciar su desarrollo.

En realidad, pues, hasta hace muy poco solamente los alcalaínos habían sostenido el proyecto de recuperar la Universidad, pero también es cierto que no tenían la capacidad de conseguirlo. La encorsetada legalidad de las cuestiones universitarias en España, reservadas casi en exclusiva al Estado, y la falta de recursos económicos en el ámbito local, impedían pensar en alguna fórmula autóctona y autónoma de universidad, como ha sido habitual en otros países. Sólo la Iglesia, es este caso la Compañía de Jesús y para formación interna, creó en 1950 una "Facultad de Filosofía", que estuvo abierta hasta 1968. 


\subsubsection{Los primeros pasos}

La Sociedad de Condueños, siendo, por una parte, fiel a sus principios constitutivos y, por otra, consciente de su incapacidad para mantener en pie los edificios que había adquirido, estuvo siempre dispuesta a ofrecer los mismos a cualquier institución que expresara el deseo de mantenerlos o recuperarlos para usos educativos. Esta fue la intención de la primera cesión en usufructo, con pago de un canon anual, que hizo del Colegio de San Ildefonso y en 1861 del de San Pedro y San Pablo a los PP. Escolapios para el establecimiento de enseñanzas medias y superiores, incluidas las preparaciones para el ingreso de escuelas especiales, entre ellas las militares. Igualmente ocurrió en 1929 con la cesión de parte del Colegio de San Jerónimo al Patronato Nacional de Turismo para "Hostería del Estudiante", que conservaría de enseñanza solamente el apellido. Durante la Segunda República, el alquiler de los Escolapios sería transferido al Estado, para el establecimiento en San Ildefonso y San Pedro y San Pablo del Instituto de Enseñanza Media Complutense.

Tras la Guerra Civil, buena parte de los edificios históricos de Alcalá estaban más arruinados que nunca. Hechos paradigmáticos de destrucción fueron los de la Iglesia de Santa María, de la Iglesia Magistral y, particularmente, el Palacio Arzobispal o Archivo General de la Administración. Pero igualmente había sufrido el patrimonio de la Sociedad de Condueños y ésta no tenía medios de restaurarlo.

Inicialmente el arrendamiento de San Pedro y San Pablo se asumió por el Ministerio de Educación Nacional, que mantuvo en él el Instituto, 
pero con respecto al edificio principal, San Ildefonso, que estaba en estado de abandono total, la Sociedad entró en negociaciones con el Estado, para que se incluyera en el catálogo a restaurar con cargo a "Regiones Devastadas" en 1947, fruto de las cuales fue la expresión de interés por parte de la Secretaría General del Movimiento de instalar en él la "Escuela de Formación Política". Ésta fue creada en 1942 con la finalidad de formar dirigentes para el nuevo régimen, y en el proyecto estaba también involucrado el Ministerio de Educación Nacional, que en ese momento era arrendatario del edificio de San Ildefonso.

La cesión al Ministerio de Educación y Ciencia se realizó en 1947, tras tensas negociaciones en las que se traslucía el recelo de la Sociedad a desprenderse de un patrimonio que fuera destinado a unos fines que, antes o después, no fueran aquellos de los que, por mandato constitutivo, era depositaria. La Sociedad hubiera preferido un arrendamiento, aunque hubiera sido simbólico, sometido a una cláusula sobre su finalidad. El Estado exigía una cesión sin más. Éste jugaba con la ventaja, no sólo de la fidelidad política contra la que en esos momentos tan peligroso resultaba no mostrar acatamiento, sino con el hecho de ser la única instancia que tenía los medios para salvar de la ruina tan preciado inmueble. El resultado, aceptado a su pesar por la Sociedad, fue una cesión completa al Estado con una cláusula que obligaba destinar el patrimonio cedido a la enseñanza superior o secundaria "católica".

De este modo el Colegio de San Ildefonso, más la capilla y sus dependencias, pasaron al Estado, afectados al Ministerio de Educación Nacional1, lo que implicó que Regiones Devastadas se hiciera cargo de las obras de restauración en 1948. En un primer momento, tanto el 
Ministro de Educación, Ibáñez Martín, como el del Movimiento, Arrese, se interesaron vivamente por las obras, a las que acudieron en algunas ocasiones, con la intención de poner en marcha lo antes posible el proyecto de formación fascista formulado en 1942.

1 Y es interesante reseñar que en el acto de recepción del citado patrimonio, que se celebró en el Paraninfo de Alcalá, firmó en representación del Ministro el rector de la Universidad Central de Madrid, con lo que había al menos un reconocimiento simbólico de los fines universitarios que se proponía dicho Ministerio con aquellos edificios.

\subsubsection{Escuela Nacional de Administración Pública}

Los cambios de matiz que hubo en el régimen franquista en 1952, con el comienzo de la marginación de las tendencias más netamente falangistas y el acceso de la tecnocracia, significó el abandono de aquel proyecto de enseñanza política; pero el Gobierno vio entonces la funcionalidad de tan formidable edificio, ya restaurado, para un nuevo y ambicioso proyecto, propio del reformismo "técnico" que se pretendía en la Administración y la economía del país: la instalación de la llamada "Escuela de la Administración Pública", que incluía entre sus organismos especializados el "Instituto de Desarrollo Económico", y que nacía de la mano de López Rodó. Una vez constituida aquélla, San Ildefonso, la capilla y demás dependencias, con la excepción del Paraninfo y el Patio Trilingüe (que quedaron en manos de Educación) fueron afectadas a Presidencia del Gobierno para el establecimiento de sus oficinas, aulas, residencia y bibliotecas.

La instalación en Alcalá de la ENAP fue un hecho importante para el futuro renacer universitario de la ciudad, no valorado todavía en sus justos términos. 
Por una parte significó la salvación y conservación, hasta hoy, del principal edificio universitario alcalaíno, que es, a su vez, uno de los testimonios vivos de la historia universitaria española.

En segundo lugar significó la reintroducción de los intereses estatales en una ciudad que entonces no iba a ser un emplazamiento de vida local, ni más adelante una simple "ciudad satélite" industrial o "dormitorio" de Madrid.

En tercer lugar, reabrió entre los círculos universitarios españoles (cuyos profesores mayoritariamente surtían, a través de cursos y seminarios, la enseñanza de la escuela) el conocimiento de esta universidad desaparecida.

Finalmente, a través de uno de sus organismos especializados, el CECA (Centro Español de Cooperación Administrativa) lanzó de nuevo al mundo iberoamericano la que se empezó a llamar "Escuela de Alcalá" uniéndola a la gran tradición que había tenido la antigua Universidad, gracias a la formación en Alcalá de cuadros para la Administración de los países de aquel área.

Otro fenómeno de consecuencias no desdeñables fue que entre los ejecutivos de dicha escuela y el personal administrativo de la misma, que significaron el primer aporte de "nuevas clases medias" a la ciudad de Alcalá, surgieron a veces conspicuos defensores de la recuperación universitaria de la ciudad complutense.

... (continuará) 